\title{
Analysis and Design of Tubular Coils for Wireless Inductive Power Transfer Systems
}

\author{
Jesús Acero ${ }^{1}$, Javier Serrano ${ }^{1}$, Claudio Carretero ${ }^{2}$, Ignacio Lope ${ }^{3}$, and José Miguel Burdío ${ }^{1}$ \\ ${ }^{1}$ Department of Electronic Engineering and Communications. Universidad de Zaragoza. \\ María de Luna 1. 50018 Zaragoza, Spain \\ 2 Department of Applied Physics. Universidad de Zaragoza. \\ Pedro Cerbuna 12. 50009 Zaragoza, Spain \\ ${ }^{3}$ BSH Home Appliances Group. \\ Avda. de la Industria, 49. 50016 Zaragoza. Spain
}

\begin{abstract}
We evaluate copper tubes as alternative of litz wire for planar coils of inductive power transfer (IPT) systems. For this purpose, a design methodology focused on maximizing the product of the magnetic coupling and the quality factor is proposed. The methodology is based on a combination of FEA simulations and analytical calculations of the skin and proximity losses in the tube. Analytical calculations are based on solutions of Maxwell's equations for a tubular geometry. These solutions are oriented to extract both skin and proximity losses in the tube. Performance of a prototype of $1.5 \mathrm{~kW}$ is evaluated and compared with a conventional litz-wire implementation.
\end{abstract}

Keywords- Analytical models, eddy currents, energy efficiency, inductive power transmission, mutual coupling.

\section{INTRODUCTION}

Copper tubes are used in many industry electrical applications at medium-high frequencies, ranging from several $\mathrm{kHz}$ up to $\mathrm{MHz}$, in order to alleviate the severe ac losses, caused by skin and proximity effects. Some popular applications of copper tubes are induction furnaces or magnetics for broadcasting systems [1,2]. Tubular conductors have some convenient mechanical and thermal properties. As examples, in some cases bobbins are unnecessary, the operation temperature of bare copper tubes is higher than the temperature of insulated magnet wires, and hollow tubes can be cooled by circulating a coolant element. Additionally, measurements presented by some authors show that at a certain frequency the stranded conductor becomes inferior to a hollow conductor [3]. Moreover, copper tubes are cheaper than other wires, especially for litz wires with high stranding level.

On the contrary, copper tubes have some disadvantages. In general, regular copper tubes are manufactured with nonelectrolytic copper and therefore its resistivity could be up to twice with respect to the copper for electrical applications. As a consequence, at the low frequency regime copper tubes have higher losses than equivalent single or multistranded conductors. However, it is interesting to note that proximity effect directly depends on resistivity [4] and therefore, depending on the frequency copper tubes can be competitive.

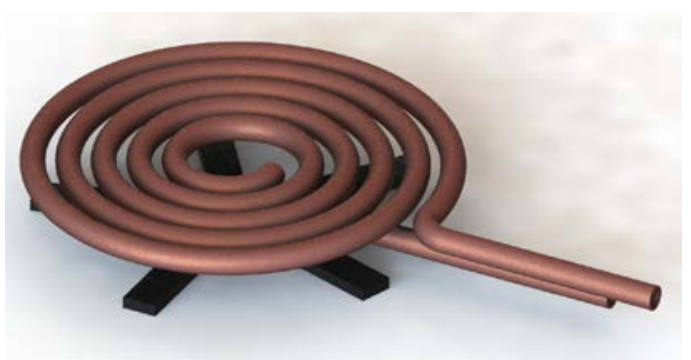

Fig. 1. Schematic representation of a power pad with tubular conductor..

Other disadvantage is that usually copper tubes are uninsulated and therefore, depending on the arrangement, insulation could be required.

In the above mentioned applications the usual shape of copper-tube inductors is solenoidal whereas planar arrangements are far less common. In this work the possibility of using copper tubes in a planar winding for inductive power transfer (IPT) systems is explored. A schematic representation of a copper-tube power pad for an inductive power transference (IPT) system is shown in Fig. 1. In this work, the analysis is focused on devices in the range of several $\mathrm{kW}$ at frequencies of tens of $\mathrm{kHz}$. For this purpose, a design procedure based on the criteria of maximum $k Q$ is explored [5-7], where $k$ is the magnetic coupling and $Q$ the quality factor. The presented procedure is aimed to obtain the number of turns and the dimensions of the tube, namely internal and external radii, for a specific application. The procedure combines FEA simulations and analytical calculations of both skin and proximity effects. The design procedure is validated by means of a prototype of $1.5 \mathrm{~kW}$. Moreover, a comparison in terms of quality factor between the designed power pad and a power pad of identical geometry with litz wire is also presented.

This paper is organized as follows. The analytical model of skin and proximity losses in copper tubes is outlined in Section II. Section III presents the design methodology and the followed optimization design process for the power pad. Experimental 
results and comparison with the litz wire are presented in Section IV. Finally, some conclusions are drawn in Section V.

\section{MOdEL OF SKIN AND PROXIMITY EFFECTS IN COPPER TUBES}

Study of IPT systems has been usually addressed by means of Finite Element Analysis (FEA) tools. Many examples of FEA analysis of IPT systems can be found in literature [8-10]. In these analysis windings are modeled as loss-free ideal domains with a constant current density and, therefore, the cabling structure is, in general, not considered. This modelling strategy reduces the computing cost but the, on the other hand, copper losses are not obtained. Some authors separately calculate the copper losses by means of a loss model of the windings with the magnetic fields obtained by simulations [11]. This is equivalent to decouple the problem and, in general, is valid if the induced currents of windings do not substantially modify the magnetic fields.

The decoupled approach is adopted in this work in order to calculate the copper-tube winding losses, which are required to estimate the quality factor of power pads. This approach makes easier an eventual optimization process because it allows to perform studies with respect to some parameters, namely number of turns or diameters of conductors, more expeditiously than FEA simulations with the actual structure of the windings.

The first step of this methodology consists of replacing the copper-tube windings by an ideal constant current density of rectangular cross section as it is schematically shown in Fig. 2. In this case, a copper-tube winding of $n$ turns whose radii range from $a_{1}$ to $a_{n}$ is considered. The geometry of the tube is represented in Fig. 3 and its external and internal radii are $r_{\text {ext }}$ and $r_{\text {int }}$, respectively. In FEA simulations the rectangular current density covers the tubular winding and therefore its height and width are $\phi_{\mathrm{ext}}=2 r_{\mathrm{ext}}$ and $\left(b_{\mathrm{ext}}-b_{\mathrm{int}}\right)$, respectively, where

$$
\begin{aligned}
& b_{\text {ext }}=a_{n}+r_{\text {ext }} \\
& b_{\text {int }}=a_{1}+r_{\text {int }}
\end{aligned}
$$

and therefore the area of the model of the winding is $S_{\text {winding }}=$ $\phi_{\text {ext }} \cdot\left(b_{\text {ext }}-b_{\text {int }}\right)$ The current density of the rectangular winding model is defined as follows:

$$
\mathbf{J}=n \frac{\hat{\imath}}{S_{\text {winding }}} \hat{\boldsymbol{\phi}}=n \frac{\hat{\imath}}{\phi_{\mathrm{ext}} \cdot\left(b_{\mathrm{ext}}-b_{\mathrm{int}}\right)} \hat{\boldsymbol{\phi}}
$$

This simulation model can also consider other elements as ferrites or shielding elements. FEA simulations of this idealized model allows to extract some interest parameters as the selfinductances or mutual inductance between windings. Moreover, the magnetic field, required to calculate the proximity effect, is also obtained in this simulations. A picture of a FEA simulation of a copper-tube power pad is shown in Fig. 4 and a FEA simulation of the equivalent current density of a power pad is shown in Fig. 5.

The second step of this method consists of including the winding losses. Skin and proximity effects of cables are included by means of the analytical model presented in this

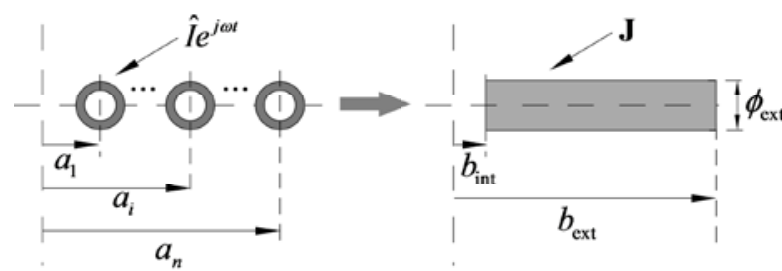

Fig. 2. FEA model of the tubular winding.

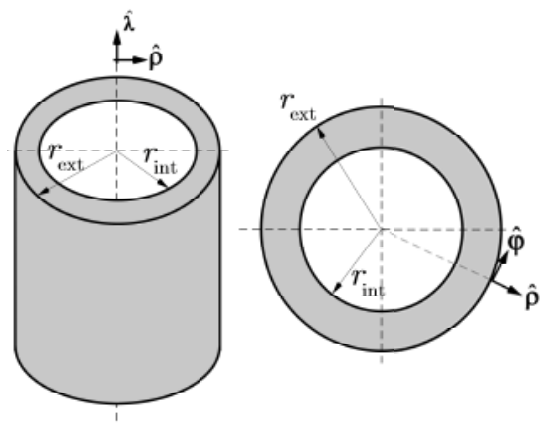

Fig. 3. Geometrical parameters of the tubular conductor.

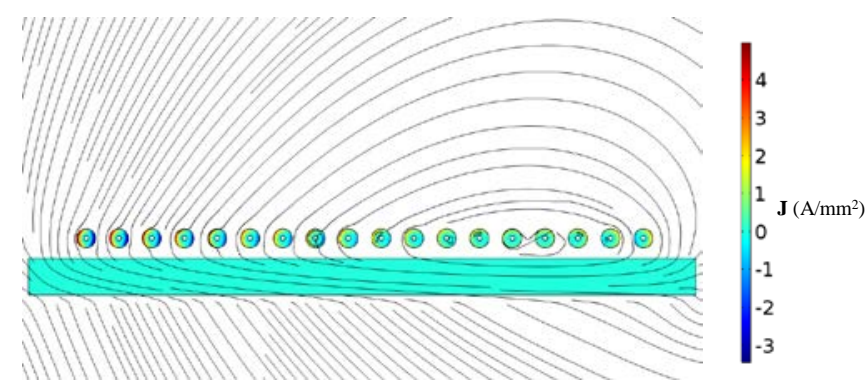

Fig. 4. FEA simulation of a power pad with tubular conductors.

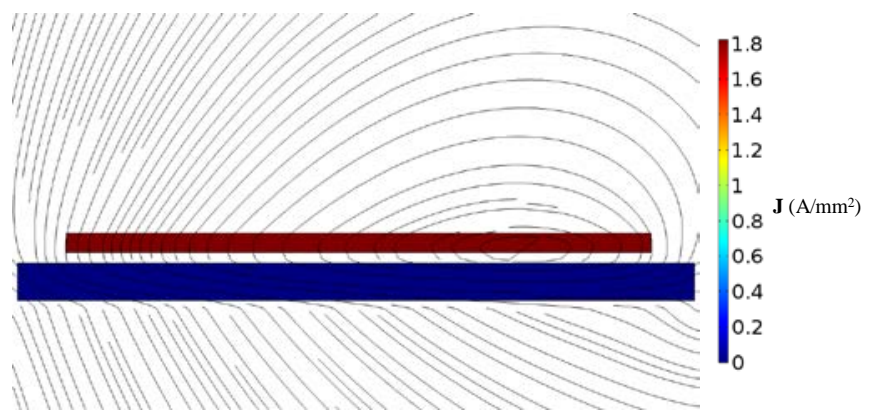

Fig. 5. FEA simulation of the equivalent current density of a power pad.

section. A model of winding resistance is proposed on the basis of losses in isolated widely-spaced tubular conductors. These losses can be caused by dc currents and ac currents, by means of skin and proximity effects. Considering that the proximity losses are induced by the winding itself, the dc, skin, and proximity losses can be associated to resistances $R_{\mathrm{dc}}, R_{\mathrm{skin}}$ and $R_{\text {prox. }}$ Therefore, the winding resistance, $R_{w}$ can be calculated as follows:

$$
R_{w}=R_{\text {dc }}+R_{\text {skin }}+R_{\text {prox }}=R_{\text {cond }}+R_{\text {prox }}
$$




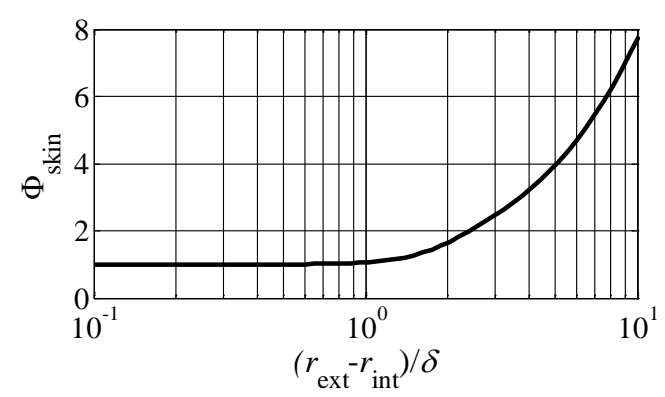

(a)

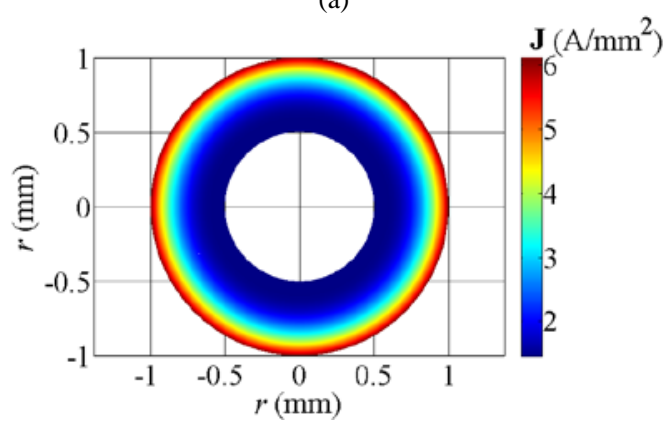

(c)

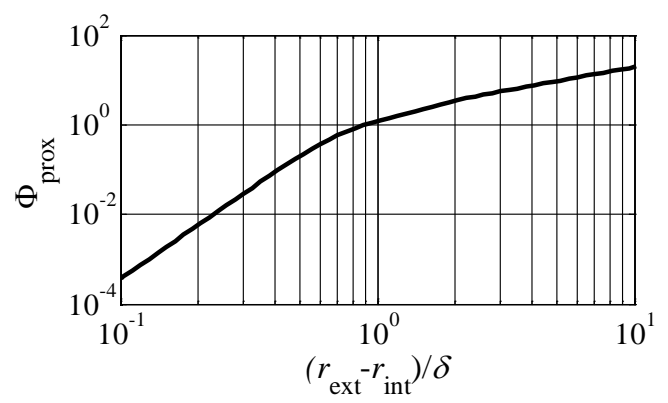

(b)

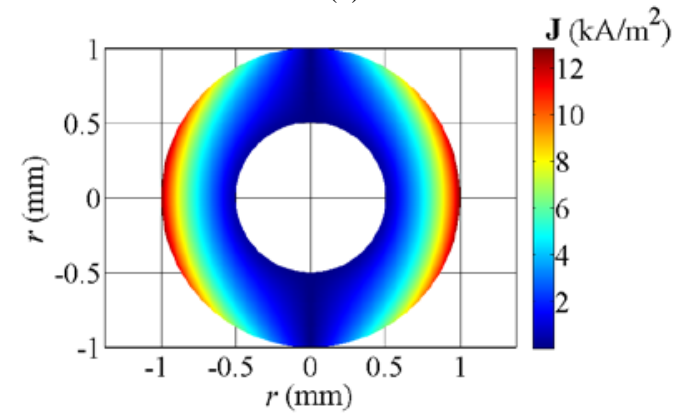

(d)

Fig. 6. (a) $\Phi_{\text {skin }}\left(r_{\text {ext }}-r_{\text {int }}\right) / \delta$. (b) $\Phi_{\text {prox }}\left(r_{\text {ext }}-r_{\text {int }}\right) / \delta$. (c) Currents in a copper tube when a voltage of $1 \mathrm{~V} / \mathrm{m}$ at $f=500 \mathrm{kHz}$ is applied. (d) Induced current in a metter of copper tube under a magnetic field of amplitude a $\mathrm{A} / \mathrm{m}$ at $f=500 \mathrm{kHz}$.

where the sum of $R_{\mathrm{dc}}$ and $R_{\text {skin }}$ is renamed as conduction resistance $R_{\text {cond }}$, or in other words, the resistance $R_{\text {cond }}$ captures both the dc resistance and the skin effect.

Taking into account that a winding can be considered as the series connection of $n$ turns of mean length MLT, $R_{\text {cond }}$ can be expressed as follows:

$$
R_{\text {cond }}=\underbrace{\frac{n \cdot \mathrm{MLT}}{\sigma_{\mathrm{Cu}} \pi\left(r_{\mathrm{ext}}^{2}-r_{\mathrm{int}}^{2}\right)}}_{R_{d c}} \underbrace{\Phi_{\text {skin }}\left(\frac{r_{\text {ext }}-r_{\text {int }}}{\delta}\right)}_{R_{\text {skin }}}
$$

where the two terms of $R_{\text {cond }}$ have been underlined. Parameters $r_{\text {ext }}$ and $r_{\text {int }}$ are represented in Fig. 3 and corresponds to the external and internal radii of the copper tube, respectively. Moreover, $\sigma_{\mathrm{Cu}}$ is the electrical conductivity of copper.

The function $\Phi_{\text {skin }}$ captures the frequency dependence of the resistance of the tube, due to the skin effect, and actually represents the ac resistance of the tube per unit of length. This function depends on the parameter $u=\left(r_{\text {ext }}-r_{\text {int }}\right) / \delta$, which physically represents the ratio of the tube thickness to the skin depth $\delta$. An analytical expression of the function $\Phi_{\text {skin }}(u)$ can be obtained in terms of Bessel functions, as it is shown at the end of this section.

Regarding proximity losses, they are caused by the currents induced in a turn due to the magnetic field generated by the neighbor turns. Considering that the proximity losses are induced by the winding itself, they can be associated to a resistance whose value is given by the following expression:

$$
R_{\text {prox }}=\frac{4 \pi}{\sigma_{\mathrm{Cu}}} \Phi_{\text {prox }}\left(\frac{r_{\text {ext }}-r_{\mathrm{int}}}{\delta}\right) \sum_{i=1}^{n}\left(2 \pi \mathrm{a}_{i}\left|\bar{H}_{i}\right|^{2}\right)
$$

where $a_{i}$ is the radius of the ith turn and $\left|\bar{H}_{i}\right|^{2}$ is the squared field on the ith turn generated by the rest of turns of the winding per unit of Ampère. The function $\Phi_{\text {prox }}(u)$ takes into account the frequency dependence of the proximity effect.

The squared field $\left|\bar{H}_{i}\right|^{2}$ not only depends on the number of turns but also depends on the presence of ferrites or other shielding elements, as aluminum plates. In that cases, the field also depends on frequency. The quantity $\left|\bar{H}_{i}\right|^{2}$ can be obtained by means of FEA either simulations or analytical calculations. In general, obtaining the field in each turn could be cumbersome. Therefore, as it was above mentioned, in this cases it could be advantageous to replace the structure of windings by an equivalent constant current density.

The field is obtained by FEA simulations with this ideal winding model, per Ampère and per turn. In this case, it is necessary to take into account that the magnetic field is proportional to the number of turns and therefore the squared magnetic field is proportional to the squared number of turns. Moreover, instead of calculating the field in each turn, the squared field is averaged in the cross sectional area of the winding. As a result (5) is rewritten as follows:

$$
R_{\text {prox }}=n^{3} \frac{4 \pi}{\sigma_{\mathrm{Cu}}} \Phi_{\text {prox }}\left(\frac{r_{\text {ext }}-r_{\text {int }}}{\delta}\right)\left\langle 2 \pi r|\bar{H}|^{2}\right\rangle_{S_{\text {winding }}}
$$


where $\left\langle 2 \pi r|\bar{H}|^{2}\right\rangle_{S_{\text {winding }}}$ is the mean value of $2 \pi r|\bar{H}|^{2}$ in the cross-sectional area of the winding, assumed as an ideal rectangular constant current density, being this value extracted from FEA simulations per Ampère and per turn.

Analytical expressions of functions $\Phi_{\text {skin }}$ and $\Phi_{\text {prox }}$ can be derived by means of the model of losses presented in [12]. In this work, an analytical model of skin and proximity losses in multilayer round conductor was reported. The functions $\Phi_{\text {skin }}$ and $\Phi_{\text {prox }}$ for copper tubes can be obtained by assuming a twolayer round conductor with the geometry presented in Fig. 1, or in other words, $\Phi_{\text {skin }}$ and $\Phi_{\text {prox }}$ can be obtained by particularizing the results of [12] to the case of two layers. These functions include several Bessel functions and can be easily implemented in Matlab.

Fig. 6(a) and (b) show the functions $\Phi_{\text {skin }}$ and $\Phi_{\text {prox }}$, respectively, with respect to the ratio $u=\left(r_{\text {ext }}-r_{\text {int }}\right) / \delta$. As occurs in solid round conductors, both skin and proximity effects become more relevant at higher frequencies, therefore, in a design, values of $\left(r_{\text {ext }}-r_{\text {int }}\right) / \delta>1$ should be avoided. On the other hand, Fig. 6(a) and (b) show the current densities due to both skin and proximity effects at $f=500 \mathrm{kHz}$ for a copper tube of radii $r_{\text {ext }}=1 \mathrm{~mm}$ and $r_{\text {int }}=0.5 \mathrm{~mm}$. The skin effect is obtained by applying a voltage of $1 \mathrm{~V}$ per unit of length, whereas the proximity effect corresponds to the currents induced in the tube by a magnetic field of a $1 \mathrm{~A} / \mathrm{m}$.

In order to compare with equivalent litz-wire windings, it also results of interest to estimate its winding resistance. In this case, expression (3) is also valid because the mechanisms that generate losses in copper tubes and litz wires are the same. Considering a litz wire of $n_{s}$ strands each one of diameter $\phi_{s}=$ $2 r_{s}$, the corresponding conduction and proximity resistances are respectively:

$$
\begin{gathered}
R_{\text {cond }}=\frac{n}{n_{s}} \frac{\text { MLT }}{\pi r_{s}^{2} \sigma_{\mathrm{Cu}}} \Phi_{\text {skin round }}\left(r_{s} / \delta\right) \\
R_{\text {prox }}=n^{3} n_{s} \frac{4 \pi}{\sigma_{\mathrm{Cu}}} \Phi_{\text {prox round }}\left(r_{s} / \delta\right)\left\langle 2 \pi r|\bar{H}|^{2}\right\rangle_{S_{\text {winding }}}
\end{gathered}
$$

These expressions are similar to (4) and (6) but (7) and (8) includes the effect of the number of strands and functions $\Phi_{\text {skin }}$ and $\Phi_{\text {prox }}$ for tubes are replaced by functions $\Phi_{\text {skin round }}$ and $\Phi_{\text {prox }}$ round for round conductors. These functions are expressed in terms of Bessel functions and corresponds to equations (9) and (10) of [13].

\section{DESIGN METHODOLOGY}

The design methodology is based on the criterion of maximum Figure of Merit (FOM) $k Q$ factor, which is defined by the product of the magnetic coupling $k$ and the inductor quality factor $Q$. References [5-7] point that the maximum efficiency of a power transmission system is:

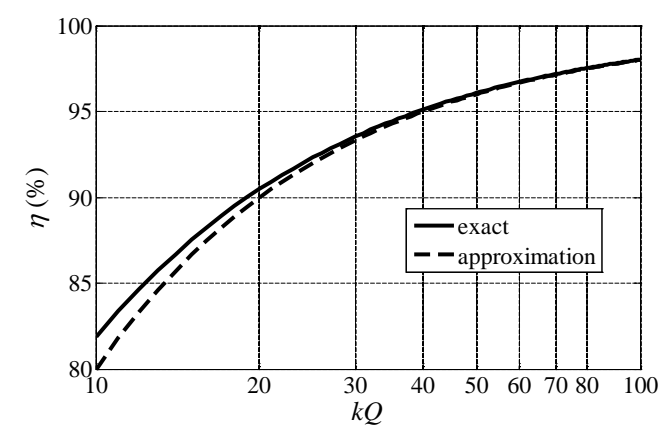

Fig. 7. Efficiency of an IPT system with respect to $k Q$ factor.

$$
\eta_{\max }=\frac{(k Q)^{2}}{\left(1+\sqrt{1+(k Q)^{2}}\right)^{2}}
$$

which usually is approximated by:

$$
\eta_{\max } \approx 1-\frac{2}{k Q}
$$

This results points that the $k Q$ factor should be maximized in order to maximize the efficiency. Fig. 7 shows the efficiency with respect to $k Q$ obtained from (9) and (10). According to this result, in order to achieve an efficiency of at least $90 \%$ the $k Q$ factor should be higher than 20 .

Considering an IPT system of two coils, the quality factor of the system is defined by the geometric average of the two individual coil quality factors:

$$
Q=\sqrt{Q_{1} Q_{2}}
$$

where:

$$
Q_{i}=\omega_{0} L_{i} / R_{w, i} \quad i=1,2
$$

The parameter $\omega_{0}$ is the angular frequency of the energy transference, $L_{i}$ is the inductance of each power pad, and $R_{w, i}$ is the winding resistance at the operating frequency. For each power pad $R_{w, i}$ is defined by (3).

Moreover, the magnetic coupling $k$ is:

$$
k=M / \sqrt{L_{1} L_{2}}
$$

where $M$ is the mutual inductance. Elements $M$ and $L_{i}$ depend on the IPT arrangement and they can be obtained by means of FEA simulations with the model presented in previous section.

For the sake of simplicity, an IPT system with two identical coils of $n$ turns is considered. Elements $M, L_{i}$ and $R_{i}$ depend on the number of turns as follows:

$$
\begin{aligned}
& M=n^{2} M_{1} \\
& L_{i}=n^{2} L_{i, 1}
\end{aligned}
$$




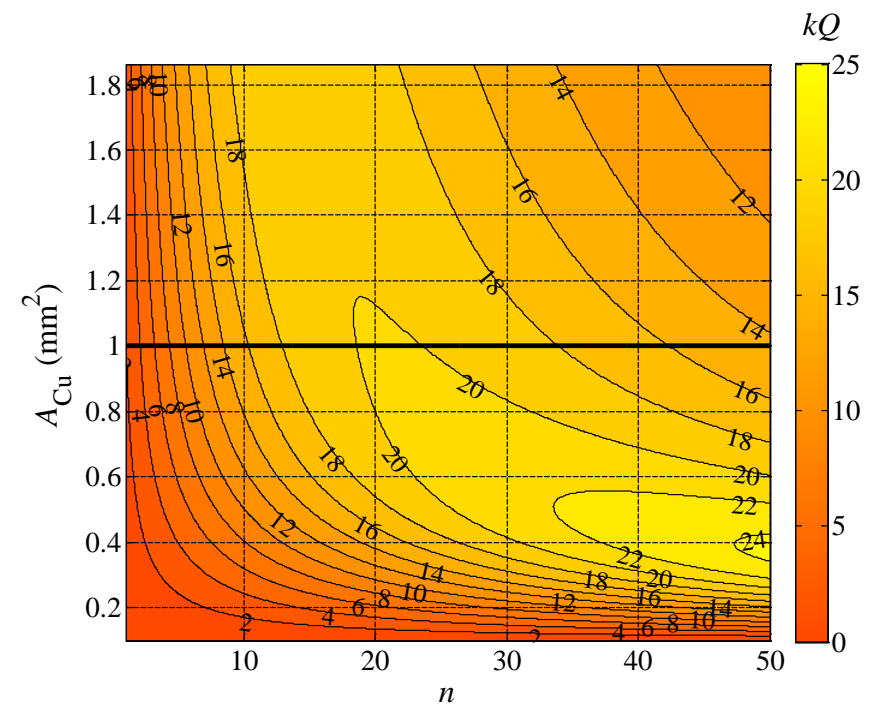

Fig. 8. Values of $k Q$ for different number of turns and cross-sectional area of the tube.

$$
R_{i}=n \cdot R_{\text {cond, } 1}+n^{3} \cdot R_{\text {prox }, 1}
$$

where $M_{1}$ is the mutual inductance between two one-turn rectangular coils of area $S_{\text {winding, }} L_{i, 1}$ is the inductance per turn of each rectangular coil, $R_{\text {cond, } 1}$ and $R_{\text {prox, } 1}$ are the conduction and proximity resistances of a one-turn winding with tubular conductor, respectively. These two values are given by the following expressions:

$$
\begin{aligned}
& R_{\text {cond }, 1}=\frac{\text { MLT }}{\sigma_{\mathrm{Cu}} \pi\left(r_{\text {ext }}^{2}-r_{\text {int }}^{2}\right)} \Phi_{\text {skin }}\left(\frac{r_{\text {ext }}-r_{\text {int }}}{\delta}\right) \\
& R_{\text {prox }, 1}=\frac{4 \pi}{\sigma_{\mathrm{Cu}}} \Phi_{\text {prox }}\left(\frac{r_{\text {ext }}-r_{\text {int }}}{\delta}\right)\left\langle 2 \pi r\left|\bar{H}_{1}\right|^{2}\right\rangle_{S_{\text {winding }}}
\end{aligned}
$$

In last equation $\left|\bar{H}_{1}\right|^{2}$ corresponds to the squared magnetic field generated by a one-turn coil. Values of $\left|\bar{H}_{1}\right|^{2}$, MLT, $M_{1}$ and $L_{i, 1}$ can be obtained from the FEA simulation of the ideal rectangular coil by adopting $n=1$.

Taking into account expressions (11) to (16), the $k Q$ factor can be rewritten as follows:

$$
\begin{aligned}
k Q & =\frac{M}{\sqrt{L_{1} L_{2}}} \sqrt{\frac{\left(\omega_{0} L_{1}\right)\left(\omega_{0} L_{2}\right)}{R_{1} R_{2}}}=\omega_{0} \cdot \frac{M}{R_{\text {cond }}+R_{\text {prox }}} \\
& =\omega_{0} \frac{n^{2} \cdot M_{1}}{n \cdot R_{\text {cond }, 1}+n \cdot n^{2} \cdot R_{\text {prox }, 1}}=\omega_{0} \frac{M_{1}}{\frac{R_{\text {cond }, 1}}{n}+n \cdot R_{\text {prox }, 1}}
\end{aligned}
$$

where it is assumed that $R_{1}=R_{2}$. For a fixed frequency, cabling structure and geometry, the solution of $\partial(k Q) / \partial n=0$ gives the number of turns $n$ which maximizes the efficiency. Manipulating in (19) it results:

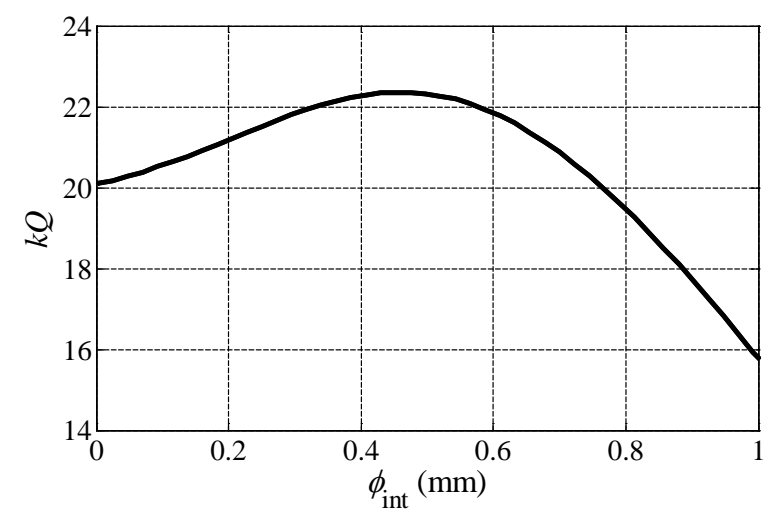

Fig. 9. Optimization of the tube dimensions at constant cross-sectional copper area.

$$
n=\sqrt{R_{\text {cond }, 1} / R_{\text {prox }, 1}}
$$

and the corresponding maximum $k Q$ factor and efficiency are:

$$
\begin{gathered}
(k Q)_{\max }=\frac{\omega_{0} M_{1}}{\sqrt{R_{\text {cond }, 1} \cdot R_{\text {prox }, 1}}} \\
\eta_{\max } \approx 1-\frac{2 \sqrt{R_{\text {cond }, 1} \cdot R_{\text {prox }, 1}}}{\omega_{0} M_{1}}
\end{gathered}
$$

The result of equation (20) is equivalent to the results obtained in other works for litz wire windings [11, 14].

Equation (22) shows that the efficiency can be improved by increasing the operating frequency, the mutual inductance or by reducing the product of the conduction by the proximity resistance. These resistances depend on the geometry of the tube and the frequency.

Despite (20) gives the theoretical number of turns which maximizes the efficiency, in practice, apart from the number of turns, the optimization of tube geometry is usually required. However, it is complicated to extract practical values from (17) and (18) because $\Phi_{\text {skin }}$ and $\Phi_{\text {prox }}$ include Bessel functions. In that case, one of the more feasible possibilities is to take approximations based on the asymptotic tendencies of $\Phi_{\text {skin }}$ and $\Phi_{\text {prox }}$ at the low-frequency range (LF):

$$
\begin{array}{cc}
\Phi_{\text {skin }}^{\mathrm{LF}}\left(\frac{r_{\text {ext }}-r_{\text {int }}}{\delta}\right)=1 & \frac{r_{\text {ext }}-r_{\text {int }}}{\delta}<1 \\
\Phi_{\text {prox }}^{\mathrm{LF}}\left(\frac{r_{\text {ext }}-r_{\text {int }}}{\delta}\right)=\frac{1}{4}\left(\frac{r_{\text {ext }}^{4}-r_{\text {int }}^{4}}{\delta^{4}}\right) & \frac{r_{\text {ext }}-r_{\text {int }}}{\delta}<1
\end{array}
$$

and therefore (17) and (18) can be rewritten as follows:

$$
R_{\text {cond }, 1}=\frac{1}{\sigma_{\mathrm{Cu}}} \frac{\text { MLT }}{\pi\left(r_{\text {ext }}^{2}-r_{\text {int }}^{2}\right)}
$$




$$
R_{\text {prox }, 1}=\frac{\pi}{\sigma_{\mathrm{Cu}}}\left(\frac{r_{\mathrm{ext}}^{4}-r_{\mathrm{int}}^{4}}{\delta^{4}}\right)\left\langle 2 \pi r\left|\bar{H}_{1}\right|^{2}\right\rangle_{S_{\text {winding }}}
$$

\section{DESIGN OF POWER PADS}

The precedent results are used to design an IPT prototype of $P=1.5 \mathrm{~kW}$ of rated power. The operating frequency is fixed at $f_{o}=100 \mathrm{kHz}$, the external diameter of power pads is $\phi_{n}=150$ $\mathrm{mm}$ and the distance between power pads is $d_{1,2}=50 \mathrm{~mm}$. Moreover, the topology of the inverter is a series resonant half bridge, the mains voltage is $230 \mathrm{~V}$ and the allowed maximum current density in windings is $J_{\max }=5 \mathrm{~A} / \mathrm{mm}^{2}$. According to these parameters, the cross-sectional area of the copper tube is set to $A_{\mathrm{Cu}}=1 \mathrm{~mm}^{2}$.

In order to increase the magnetic coupling, it is planned to include some ferrite bars below the windings. These bars are modelled as a ferrite disk with an equivalent magnetic permeability. As a result, the obtained magnetic coupling is $k=$ 0.23 .

Considering these conditions, the design process consists of determining the number of turns and the geometry of the conductors which maximizes the $k Q$ factor. For this purposes, an optimization process by means of (19) is followed. The $k Q$ factor is calculated with respect to the tube cross-sectional area and considering the number of turns as a parameter. In this case, volume restrictions are not considered in the design process, however final designs are checked in order to verify if they fit in the available space.

Fig. 8 (a) shows the calculated $k Q$ values for different number of turns and copper areas. In this figure the limit of $A_{\mathrm{Cu}}$ is also represented. According to these results, the maximum $k Q$ is comprised between 19 and 23 turns. Therefore, considering $n=19$ turns an optimization of the tube geometry is further explored. For this purposes, the $k Q$ factor is calculated for different values of the internal and external diameters of the tube when constant copper area of $A_{\mathrm{Cu}}=1 \mathrm{~mm}^{2}$ is considered.

Fig. 9 shows the values of $k Q$ for different internal diameter of the copper tube. Considering these results, the selected internal and external diameters of the tube are $\phi_{\mathrm{ext}}=1.8 \mathrm{~mm}$, $\phi_{\text {int }}=0.45 \mathrm{~mm}$.

\section{EXPERIMENTAL RESULTS}

A handmade prototype was built by using a plastic holder with the selected copper tube and the tested power pad is shown in Fig. 10.

Experimental verification is mainly intended for checking the accuracy of the previously presented design methodology and calculations. Taking into account that in an IPT system the coupling factor is defined by the geometrical arrangement, the experimental verification can be focused on the quality factor whereas the coupling factor is obtained from simulations.

The measured and calculated values of the $k Q$ factor are shown in Fig. 11. Experimental and calculated results show good concordance. Some discrepancies are observed at the high frequency range due to the significance of the parasitic capacitance of the winding, which is not included in the model.

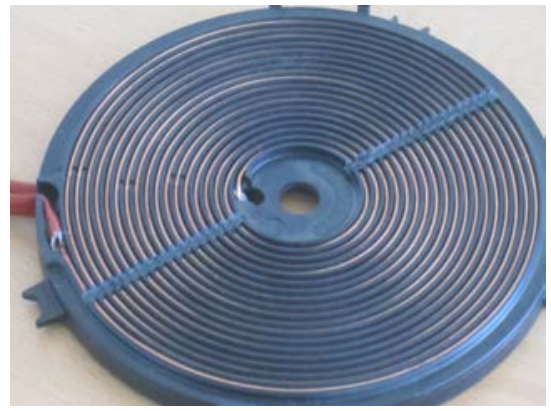

Fig. 10. Picture of the power pad prototype.

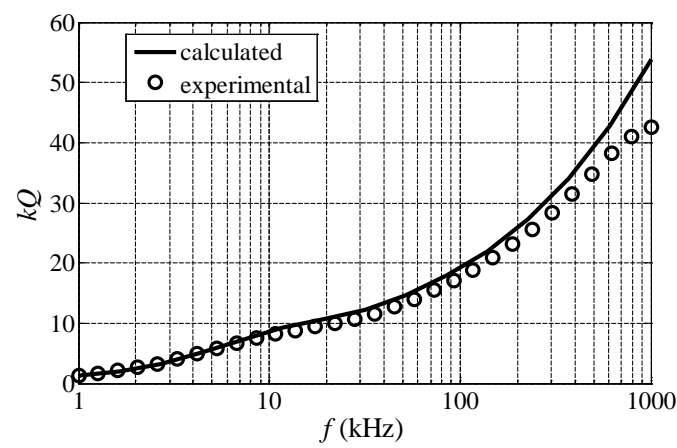

Fig. 11. Comparison between calculated and experimental $k Q$ factors.

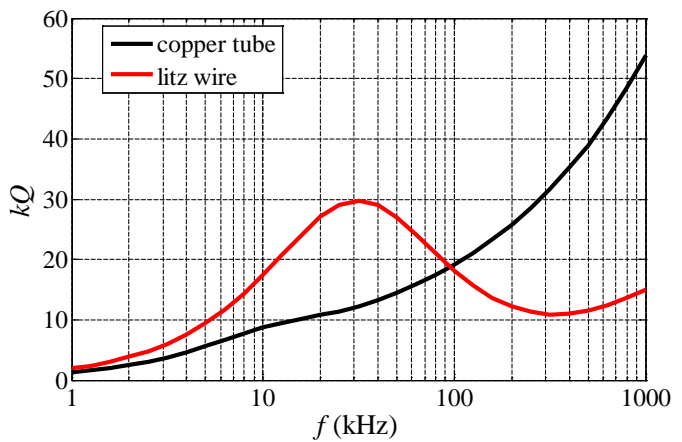

Fig. 12. Comparison between copper tube and litz wire $k Q$ factors of power pads.

Additionally, this design is compared with a conventional litz-wire power pad. In this case, only calculated results are compared because the litz-wire prototype was not available. The power pad has the same number of turns and the simulated litz wire has $n_{s}=19$ copper strands of $\phi_{s}=400 \mu \mathrm{m}$. Therefore, the cross-sectional area is also the same in both cases. $k Q$ factors for both designs with respect to the frequency are presented in Fig. 12. Results show that, apart from cost, the copper tubes could be competitive at frequencies higher than $100 \mathrm{kHz}$.

\section{CONCLUSION}

Copper tubes are evaluated for applications at medium-high frequencies in the range of kilowatts. A design methodology oriented to optimize the parameter $k Q$ is proposed. Methodology is based on a combination of FEA idealized simulations and analytical calculations of the resistance in the 
windings and therefore studies with respect to the number of turns and the geometry of the conductors become affordable. The methodology has been tested by means of impedance measurements on a prototype. The proposed arrangement compared with a conventional litz-wire power pad presents superior performance at frequencies above $100 \mathrm{kHz}$.

\section{ACKNOWLEDGMENT}

This work was partly supported by the Spanish MINECO under Project TEC2013-42937-R, Project CSD2009-00046, and Project RTC-2014-1847-6, by the DGA-FSE, by the University of Zaragoza under Project JIUZ-2015-TEC-12, and by the BSH Home Appliances Group.

\section{REFERENCES}

[1] G. H. Brown, C. H. Hoyler, and R. A. Bierwirth, Theory and Application of Radio-Frequency Heating. New York, NY, USA, 1947.

[2] J. Davies, Conduction and Induction Heating. Stevenage, U.K., 1990.

[3] J. A. Ferreira, "Analytical computation of AC resistance of round and rectangular litz wire windings," IEE Proceedings B, Electric Power Applications, vol. 139, pp. 21-25, 1992.

[4] J. A. Ferreira, Electromagnetic Modelling of Power Electronics converters. Norwell, MA: Kluwer, 1989.

[5] R. Bosshard, J.Mühlethaler, J. W. Kolar, and I. Stevanovi, "Optimized magnetic design for inductive power transfer coils," presented at the The 28th Annual IEEE Applied Power Electronics Conference and Exposition (APEC), 2013, 2013.
[6] O. Knecht, R. Bosshard, and J. W. Kolar, "High-efficiency transcutaneous energy transfer for implantable mechanical heart support systems," IEEE Transactions on Power Electronics, vol. 30, pp. 6221-6236, Nov. 2015.

[7] G. Vandevoorde and R. Puers, "Wireless energy tranfer for stand-alone systems: A comparison between low and high power applicability" in Sensors and Actuators A: Physical vol. 92, ed, 2001, pp. 305-311.

[8] J. Zhang, X. Yuan, C. Wang, and Y. He, "Comparative analysis of twocoil and three-coil structures for wireless power transfer," IEEE Transactions on Power Electronics, vol. 32, pp. 341-352, Jan. 2017.

[9] Z.-H. Ye, Y. Sun, X. Dai, C.-S. Tang, Z.-H. Wang, and Y.-G. Su, "Energy efficiency analysis of U-coil wireless power transfer system," IEEE Transactions on Power Electronics, vol. 31, pp. 4809-4817, Jul. 2016.

[10] F. Y. Lin, G. A. Covic, and J. T. Boys, "Evaluation of magnetic pad sizes and topologies for electric vehicle charging," IEEE Transactions on Power Electronics, vol. 30, pp. 6391-6407, Nov. 2015.

[11] I. Lope, J. Acero, and C. Carretero, "Analysis and optimization of the efficiency of induction heating applications with litz-wire planar and solenoidal coils," IEEE Transactions on Power Electronics, vol. 31, pp. 5089-5101, Jul. 2016.

[12] J. Acero, C. Carretero, I. Lope, R. Alonso, and J. M. Burdío, "Analytical solution of the induced currents in multilayer cylindrical conductors under external electromagnetic sources," Applied Mathematical Modelling, Aug. 2016.

[13] J. Acero, C. Carretero, R. Alonso, and J. M. Burdío, "Quantitative evaluation of induction efficiency in domestic induction heating applications," IEEE Trans. on Magnetics, vol. 49, pp. 1382-1389, Apr. 2013.

[14] C. R. Sullivan, "Optimal choice for number of strands in a litz-wire transformer winding," IEEE Transactions on Power Electronics, vol. 14, pp. 283-291, Mar. 1999. 